\title{
Follow-up after bariatric surgery: are we effective enough?
}

\author{
Kamil Nurczyk, Chia-En Chan, Tomasz Skoczylas, Grzegorz Wallner \\ $2^{\text {nd }}$ Department of General Surgery, Medical University of Lublin, Lublin, Poland
}

Videosurgery Miniinv 2022; 17 (2): 299-302

DOI: https://doi.org/10.5114/wiitm.2021.110411

\begin{abstract}
Over the years, the rise in the obesity epidemic has led to an increasing demand for bariatric surgery. Considering the rapidly growing number of bariatric surgery procedures performed, intensive development of postoperative care and surveillance programs should be expected. However, the effectiveness of follow-up after bariatric surgery appears to be surprisingly low. The purpose of this review is to draw attention to the quality of follow-up programs and to encourage health care providers to make efforts to ensure adequate post-operative data collection. Awareness should be raised about inadequate data collection to strengthen the credibility and authenticity of treatment results, thus providing a clearer picture of treatment efficacy.
\end{abstract}

Key words: obesity, bariatric surgery, weight loss, follow-up.

\section{Introduction}

Weight loss surgery is considered a highly effective treatment method for morbid obesity. In the last decade, bariatric surgery has gained popularity each year. According to the estimates of the American Society of Metabolic and Bariatric Surgery (ASMBS), the annual number of weight-loss procedures increased from 158,000 in 2011 to 252,000 in 2018 [1], and $1,343,000$ bariatric procedures were performed in the US alone during this period (Figure 1).

The most commonly used procedures in modern bariatric surgery include: laparoscopic sleeve gastrectomy (LSG) [2], laparoscopic Roux-en-Y gastric bypass (LRYGB) [3], laparoscopic duodenal switch (LDS) [4], and laparoscopic adjustable gastric banding (LAGB) [5]. The recent development of weightloss surgery in the US is characterized by a rapid increase in the popularity of LSG, a noticeable decrease in the number of LRYGB procedures and a dramatic decrease in the number of gastric banding procedures (Figures 1 or 2).
It has been proven that weight loss surgery can not only facilitate the reduction of body mass index (BMI) but also has a positive influence on patient comorbidities, thus extending life expectancy. However, this is no one-size-fits-all solution. Schauer et al. [6] in their analysis of the impact of bariatric surgery on severely obese patients with diabetes noted that bariatric surgery seems to improve the life expectancy of most patients, but has a negative impact on patients with a BMI above $62 \mathrm{~kg} / \mathrm{m}^{2}$.

\section{Aim}

Given the huge influence of bariatric treatment on metabolism and nutrition, it is natural to expect that efficacious postoperative care and constructive follow-up programs should be provided. In support of this statement, it is worth mentioning the British National Institute for Clinical Excellence (NICE) Obesity Guidelines [7] from 2014, which emphasized the necessity of a 2-year follow-up in bariatric surgical treatment to ensure patient safety through regular assessment of diet, comorbidities, medication, as 


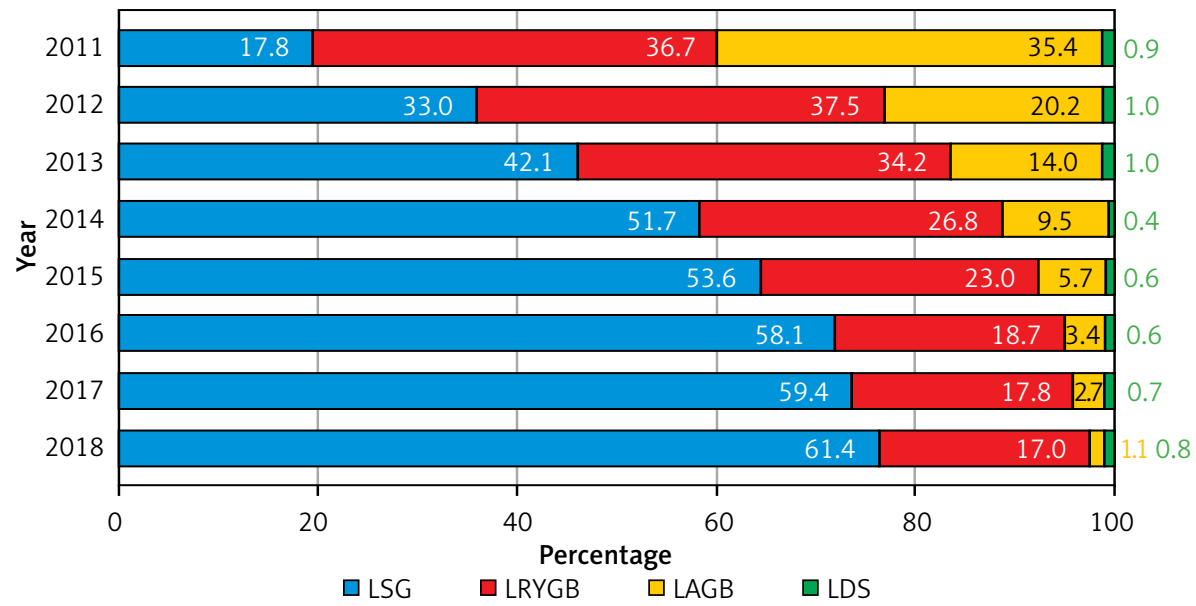

Figure 1. Annual number of bariatric surgical procedures in the US from 2011 to 2018 according to the American Society of Metabolic and Bariatric Surgery estimates

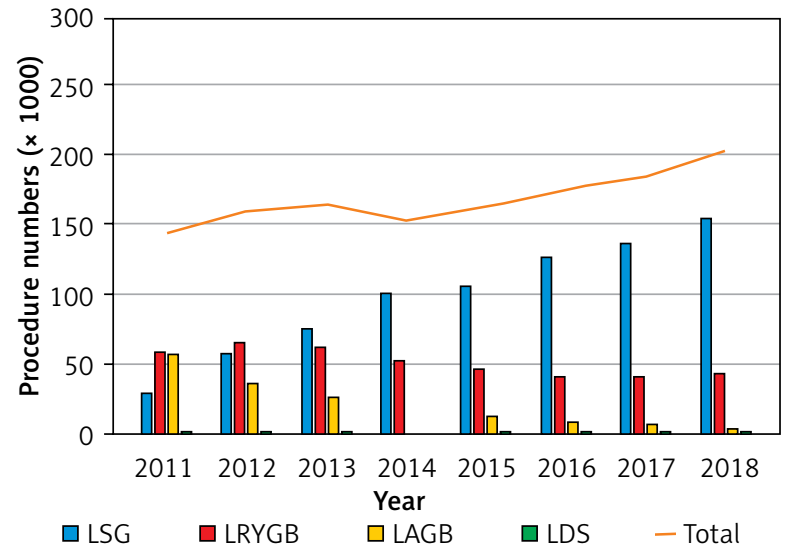

Figure 2. Annual percentage of specific bariatric procedures in the US from 2011 to 2018 according to American Society of Metabolic and Bariatric Surgery estimates

well as physical and psychological activity. Same recommendations are also encouraged by local expert groups such as the Association of Polish Surgeons [8]. The purpose of this review is to draw attention to the quality of follow-up programs and to encourage health care providers to make efforts to ensure adequate post-operative data collection.

\section{Material and methods}

A review of the English language literature was performed to identify studies and reports describing the quality of follow-up in patients who underwent bariatric surgery procedures. We used Medical Literature Analysis and Retrieval System Online (Medline), Excerpta Medica Database (EMBASE), and Cochrane Central Register of Controlled Trials (CENTRAL) and other sources if relevant. Search strategies were created using a combination of MeSH terms, subject headings and keywords to obtain the maximum number of articles.

\section{Results}

Considering the significant medical impact of weight-loss surgery, the estimated bariatric surgery follow-up rates appeared to be surprisingly low. The Fourth IFSO Global Registry Report [9] from 2018 provided the data of follow-up regarding weight loss and treatment of diabetes type 2 after bariatric surgery. In this report, all patients treated prior to 2017 were eligible for follow-up, but within 12 months after surgery, only about $40 \%$ of patients had weight change data documented, and only $30 \%$ had data on diabetes treatment collected.

Even more intriguing, although LSG is the most popular surgical treatment option, the percentage of follow-up data collected after this procedure has significantly decreased compared with patients after LRYGB. According to this report, from 2012 to 2016 the percentage of weight loss data collection over a 1-year follow-up period ranged annually from $23.8 \%$ to $33.7 \%$ for LSG compared to $54.4-59.4 \%$ for RYGB. Data concerning the treatment of diabetes type 2 were $11.6-18.6 \%$ for SG compared to $47.4-$ $52.7 \%$ for LRYGB. Although these values are highly unsatisfactory in the 1-year follow-up, they get worse on a 2-year basis because less than 1 in 10 patients had follow-up data collected at that time. 
Ensuring a long-term follow-up seems to be of the utmost importance for bariatric patients. Moreover, it is not an easy and one-way process. The necessity of controlling diabetes treatment and dietary recommendations is widely known. However, it should not be forgotten that the consequences of the bariatric surgery bring the need to monitor a wide range of factors, such as the psychological aspect, the level of physical activity [10], and even the risk assessment of a planned pregnancy [11].

Low quality follow-up in bariatric surgery is indicated not only as a risk factor for poor outcomes [12] but the versatility and effectiveness of follow-up are also determinants of clinical research, giving credibility to the results obtained. One of the conclusions of the Cochrane review by Colquitt et al. [13], which included 26 studies evaluating the effects of bariatric surgery, was that the poor quality of research should result in caution in interpreting the results. Inadequate quality of follow-up was indicated in a systematic review published in JAMA in 2014 [14]. In 2016, Switzer et al. [15] in an analysis of 99 bariatric surgery studies published between 2007 and 2012 found that only $40.4 \%$ of papers had adequate patient follow-up meeting the McMaster criteria, $42.4 \%$ had insufficient follow-up, and $17.2 \%$ reported no follow-up at all.

\section{Discussion}

The main difficulty to overcome in the case of poor follow-up is the complexity of the underlying causes. Several factors have already been described, and they include economic barriers, long distance to travel and language barriers. To decrease the number of patients lost to follow-up, the importance of post-operative visits should be widely discussed with the patient during the pre-operative work-up and education time. More opportunities for improvement may lie in changing the operation of clinics so as to eliminate long waiting times and inconvenient appointment hours. There is a variety of strategies implemented in many centers such as flexible appointment dates, appointment reminders by phone call, registries of missed appointments, and using different ways for reaching patients.

In their randomized studies, both Peterli et al. [16] and Salminen et al. [17] compared the results of LSG and LRYGB. By obtaining data from 5-year follow-up, in addition to the valuable conclusions that these two procedures are comparable in terms of weight loss, they also confirmed that LSG is associated with an increased risk of gastroesophageal reflux symptoms. Naturally, these findings were a result of longterm data collection. In our opinion, only thorough, tedious and universal follow-up data collection will give an ability to directly assess long-term outcomes for different procedures and patient groups. We do believe that bringing follow-up to a higher level can only assure that the benefits of bariatric surgery outweigh the risks and are cost-effective.

\section{Conclusions}

Bariatric procedures, especially those that affect absorption, have a significant impact on nutritional intake. Supervision of the treatment process after surgery is the key to achieve optimal outcomes in terms of weight loss, proper nutrition and screening for complications. Failure to comply with the follow-up schedule may increase the risk of complications and reduce the long-term effect of treatment. Low effectiveness of follow-up also means that the amount of data that could help evaluate outcomes and risks of different bariatric treatment modalities is limited.

Being aware of the available space for improvements, health care professionals organized in bariatric teams should make more effort to improve the follow-up of bariatric patients to ensure the best clinical effect. We should strive to grant patients easy access to appropriate care and encourage them to play an active role in this process. Improvements in follow-up may also allow bariatric surgery studies to obtain data that genuinely reflect the features of this treatment.

\section{Conflict of interest}

The authors declare no conflict of interest.

\section{References}

1. American Society of Metabolic and Bariatric Surgery, Estimate of Bariatric Surgery Numbers, 2011-2018, June 2018. [WWW document]. URL https://asmbs.org/resources/estimate-of-bariatric-surgery-numbers

2. Palermo M, Serra E. Laparoscopic sleeve gastrectomy: how do I do it. J Laparoendosc Adv Surg Tech A 2020; 30: 2-5.

3. Nurczyk K, Herbella FA, Patti MG. Roux-en-Y gastric bypass for obesity. How we do it. J Laparoendosc Adv Surg Tech A 2020; 30: 623-6.

4. Ng PC, Sharp LS, Bermudez DM. Duodenal switch: fully stapled technique. Surg Obes Relat Dis 2019; 15: 512. 
5. Ren CJ, Fielding GA. Laparoscopic adjustable gastric banding [Lap-Band]. Curr Surg 2003; 60: 30-3.

6. Schauer DP, Arterburn DE, Livingston EH, et al. Impact of bariatric surgery on life expectancy in severely obese patients with diabetes: a decision analysis. Ann Surg 2015; 261: 914-9.

7. National Institute for Health and Care Excellence (NICE). Clinical guideline 189 - Obesity: identification, assessment and management of overweight and obesity in children, young people and adults. National Institute for Health and Care Excellence, London, 2014. [WWW document]. URL https://www.nice. org.uk/guidance/cg189/resources/obesity-identification-assessment-and-management-pdf-35109821097925.

8. Szeliga J, Wyleżoł M, Major P, et al. Metabolic and Bariatric Surgery Chapter of the Association of Polish Surgeons. Bariatric and metabolic surgery care standards. Videosurgery Miniinv 2020; 15: 391-4.

9. Himpens J, Ramos A, Welbourn R, et al. Fourth IFSO Global Registry Report 2018. Dendrite Clinical Systems Ltd, Henley-onThames, RG9 1AY, UKISBN 978-0-9929942-7-3 2018.

10. Pekař M, Pekařová A, Bužga M, et al. The risk of sarcopenia 24 months after bariatric surgery - assessment by dual energy X-ray absorptiometry (DEXA): a prospective study. Videosurgery Miniinv 2020; 15: 583-7.

11. Różańska-Walędziak A, Bartnik P, Kacperczyk-Bartnik J, et al. Pregnancy after bariatric surgery - a narrative literature review. Videosurgery Miniinv 2021; 16: 30-7.

12. Strusberg I, Bertoli AM, Ramos M, et al. Factors associated with patients' loss to follow-up after finishing randomized clinical trial participation. Contemp Clin Trials 2005; 26: 38-44.

13. Colquitt JL, Picot J, Loveman E, et al. Surgery for obesity. Cochrane Database Syst Rev 2009; 2: CD003641.

14. Puzziferri N, Roshek IIIrd TB, Mayo HG, et al. Long-term follow-up after bariatric surgery: a systematic review. JAMA 2014; 312: 934-42.

15. Switzer NJ, Merani S, Skubleny D, et al. Quality of follow-up: systematic review of the research in bariatric surgery. Ann Surg 2016; 263: 875-80.

16. Peterli R, Wölnerhanssen BK, Peters T, et al. Effect of laparoscopic sleeve gastrectomy vs laparoscopic Roux-en-Y gastric bypass on weight loss in patients with morbid obesity: the SMBOSS randomized clinical trial. JAMA 2018; 319: 255-65.

17. Salminen P, Helmiö M, Ovaska J, et al. Effect of laparoscopic sleeve gastrectomy vs laparoscopic Roux-en-Y gastric bypass on weight loss at 5 years among patients with morbid obesity: the SLEEVEPASS randomized clinical trial. JAMA 2018; 319: 241-54.

Received: 4.07.2021, accepted: 6.09.2021. 